\title{
Coronal Magnetic Fields of Algol Binaries from Microwave Spectra
}

\author{
G. Umana ${ }^{1}$, C. Trigilio ${ }^{1}$, R. M. Hjellming ${ }^{2}$, S. Catalano ${ }^{3}$, \\ M. Rodonò ${ }^{3,4}$ \\ ${ }^{1}$ Istituto di Radioastronomia CNR, stazione di Noto, C.P. 169, Noto, Italy \\ ${ }^{2}$ National Radio Astronomy Observatory, Socorro, N. M., USA \\ ${ }^{3}$ Istituto di Astronomia, Università di Catania, V. A. Doria 6, \\ 95125 Catania, Italy \\ ${ }^{4}$ Osservatorio Astrofisico, Università di Catania, V. A. Doria 6, \\ 95125 Catania, Italy
}

\section{Introduction}

Algol-type binaries are basically known to undergo hydrodynamic processes related to mass exchange between components. Recent observations on radio, $\mathrm{X}$-ray emission and flare-like events have raised the question of possible magnetic activity in the secondary component of these systems (Hall, 1989).

From a microwave emission survey we have shown that the radio emission from Algol systems cannot be accounted for by thermal emission from an hot corona $\left(T \geq 10^{7} K\right)$ and that their radio luminosities compare very well with those of the magnetically active RS CVn systems (Umana et al., 1990).

\section{Observations and results}

Radio spectra of a small sample of Algol-type binary systems at four wavelengths $(20,6,3.6$, and $2 \mathrm{~cm})$ were obtained with the VLA in two different observing runs (18 Feb. and $6 \mathrm{Mar} .1989$ ). As in the case of RS CVn-type binary systems, the radio emission seems to be due to the interaction of mildly relativistic electron with the magnetic field of the active component (gyrosynchrotron emission). Calculations for an homogeneous structure, such as a corona surrounding the secondary component with uniform magnetic field strength and uniform density of the energetic particles, lead to spectra that are inconsistent with the shape of the observed 
spectra, for each choice of parameters (magnetic field strength, particles energy spectrum and density).

Instead, a core-halo structure for the radio sources with a compact core, with uniform magnetic field strength and density of energetic particles, together with an extended halo, with a radially decreasing particle density and a constant magnetic field strength, is able to reproduce the observed characteristics of the radio spectra.

In Table a summary of the parameters derived for the two regions for the observed systems is given. The average magnetic field strengths we derive are $80-100$ Gauss for the core and 10-20 Gauss for the halo with typical electron densities of $5-10 \times 10^{6} \mathrm{~cm}^{-3}$ and $2-5 \times 10^{6} \mathrm{~cm}^{-3}$ for the core and the halo respectively. Characteristic sizes of the core are comparable with the size of the secondary component while those of the halo turn out to be as large as the whole system.

The good agreement between the two spectra observed at two different epochs of the binary system HR 5110 and the computed gyrosynchrotron spectra, assuming a core-halo structure for the radio source (thick line), is shown in Fig. 1, as an example of our results. The error bar represents the rms on the maps plus the error in the flux determination. The dashed lines represent the different contributions to the total spectrum from the halo (low frequencies) and from the core (high frequencies).

The 6 Mar 1989 spectrum of HR 5110 is consistent with an ejection of energetic particles in the lower corona (the total number of energetic particles has increased with respect to $18 \mathrm{Feb} 1989$, see Table).

\begin{tabular}{|c|c|c|c|c|}
\hline Component & $\begin{array}{l}\text { Magnetic } \\
\text { Field } \\
\text { (Gauas) }\end{array}$ & $\begin{array}{c}\text { Linours } \\
\text { Dimension } \\
(\mathrm{cm})\end{array}$ & $\begin{array}{l}\text { Particle } \\
\text { Denalty } \\
\mathrm{cm}^{-3}\end{array}$ & $\begin{array}{c}\alpha \text { Index } \\
N(r) \propto N_{O}\left(h_{R}\right)^{\alpha}\end{array}$ \\
\hline Core & 60 & $\underset{8 \times 10^{10}}{\text { Algol }}$ & $10^{8}$ & 0 \\
\hline Halo & 20 & $4 \times 10^{11}$ & $5 \times 10^{8}$ & 2 \\
\hline Core & 40 & $\begin{array}{l}V 606 \mathrm{Sgr} \\
1.8 \times 10^{11}\end{array}$ & $2 \times 10^{6}$ & 0 \\
\hline Halo & 10 & $7 \times 10^{11}$ & $5 \times 10^{5}$ & 2 \\
\hline $\begin{array}{l}\text { Core } \\
\text { Halo }\end{array}$ & $\begin{array}{l}80 \\
40\end{array}$ & $\begin{array}{c}\text { HR } 5110 \text { (18 Feb) } \\
1.2 \times 10^{10} \\
7 \times 10^{11}\end{array}$ & $\begin{array}{l}6 \times 10^{8} \\
3 \times 10^{8}\end{array}$ & $\begin{array}{l}0 \\
2\end{array}$ \\
\hline $\begin{array}{l}\text { Core } \\
\text { Helo }\end{array}$ & $\begin{array}{l}60 \\
40\end{array}$ & $\begin{array}{c}\text { HR. } 5110 \text { (6 Mar.) } \\
1.8 \times 10^{10} \\
7 \times 10^{11}\end{array}$ & $\begin{array}{l}10^{7} \\
10^{7}\end{array}$ & $\stackrel{0}{2}$ \\
\hline $\begin{array}{l}\text { Core } \\
\text { Halo }\end{array}$ & $\begin{array}{l}100 \\
10\end{array}$ & $\begin{array}{c}\text { RZ Cas (18 Feb.) } \\
6.8 \times 10^{10} \\
7 \times 10^{11}\end{array}$ & $\begin{array}{c}10^{6} \\
3 \times 10^{5}\end{array}$ & $\begin{array}{l}0 \\
2\end{array}$ \\
\hline & & RZ Cal (6 Mar.) & & \\
\hline $\begin{array}{l}\text { Core } \\
\text { Halo }\end{array}$ & $\begin{array}{l}80 \\
10\end{array}$ & $\begin{array}{l}7 \times 10^{10} \\
7 \times 10^{11}\end{array}$ & $\begin{array}{l}7 \times 10^{5} \\
1.5 \times 10^{5}\end{array}$ & $\begin{array}{l}0 \\
2\end{array}$ \\
\hline & & TW Dra (18 Feb.) & & \\
\hline Core & 80 & $4.8 \times 10^{10}$ & $2 \times 10^{7}$ & 0 \\
\hline Halo & 10 & $3.2 \times 10^{11}$ & $4 \times 10^{6}$ & 2 \\
\hline & & TW Dra (6 Mar) & & \\
\hline $\begin{array}{l}\text { Core } \\
\text { Halo }\end{array}$ & $\begin{array}{l}80 \\
10\end{array}$ & $\begin{array}{l}8.0 \times 10^{10} \\
3.2 \times 10^{11}\end{array}$ & $\begin{array}{l}1.5 \times 10^{6} \\
4 \times 10^{6}\end{array}$ & $\begin{array}{l}0 \\
2\end{array}$ \\
\hline
\end{tabular}



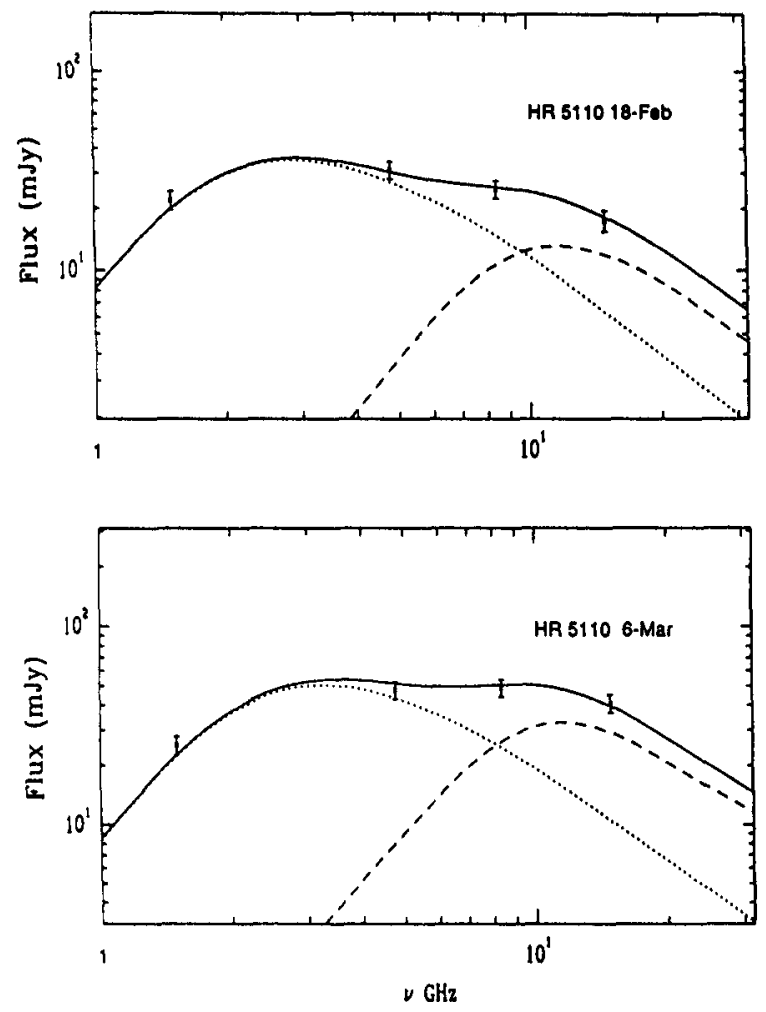

Fig. 1. Comparison between the observed radio spectra of the binary system HR 5110 and the computed spectrum obtained assuming a core-halo structure for the radio source as described in the text.

The proposed core-halo structure has not to be assumed as a rigorous model of the radio source, but it means that the probably complex topology of the coronal magnetic fields could be reduced to two basic magnetic structures: small, compact loops with high values of magnetic field strengths that we have described as the core, and larger loops, extending to the dimension of the whole systems, that we have described as the halo.

Acknowledgements. The Very Large Array (VLA) is a facility of the National Radio Astronomy Observatory operated by the Associated Universities, Inc., under contract with the National Science Foundation. This work has been supported by the CNR, the M.U.R.S. through the University of Catania, the Osservatorio Astrofisico di Catania and the GNA(CNR). The extensive use of the computer facilities of the Catania ASTRONET site is also acknowledged.

\section{References}

Hall, D. S.: 1989, Space Sci. Rev. 50, 219

Umana G., Catalano S., Rodonó M.: 1990, Astron. Astrophys., submitted 